\title{
A VARIANT OF THE INEQUALITY GIVEN BY BAINOV AND SIMEONOV
}

\author{
CHYNG NAN CHOU AND GOU-SHENG YANG
}

\begin{abstract}
In this paper explicit bounds on an integral inequality and its discrete analogue are established. These inequalities can be used as ready tools in certain applications.
\end{abstract}

\section{Introduction}

It is well known that the inequalities which yield explicit bounds on unknown functions play a fundamental role in the development of the theory of differential, integral and finite difference equations, see $[1,2,3]$. In $[1$, p.11] Bainov and Simeonov proved the following useful integral inequality:

Lemma BS. Let $U(t), a(t), b(t)$ be continuous functions on $I=[\alpha, \beta]$, let $a(t)$ and $b(t)$ be nonnegative in $I$, and suppose

$$
U(t) \leq c+\int_{\alpha}^{t} a(s) U(s) d s+\int_{\alpha}^{\beta} b(s) U(s) d s
$$

for $t \in I$, where $c$ is a constant. If

$$
q=\int_{\alpha}^{\beta} b(s) \exp \left(\int_{\alpha}^{s} a(\sigma) d \sigma\right) d s<1,
$$

then

$$
U(t) \leq \frac{c}{1-q} \exp \left(\int_{\alpha}^{t} a(s) d s\right)
$$

for $t \in I$.

The main purpose of this paper is to establish a useful variant of the above inequality which can be used as ready tool to study the qualitative behavior of solutions of certain integral equations. The discrete analogue of the main result is also given.

\section{Main Result}

In what follows, $R$ denotes the set of real numbers, $R_{+}=[0, \infty), I=[\alpha, \beta]$ are the given subsets of $R$ and $Z$ be the set of integers. For $\alpha, \beta \in Z, \alpha \leq \beta$, let $N_{\alpha, \beta}=\{n \in Z$ :

Received August 9, 2004; revised November 4, 2004.

Key words and phrases. Bainov, Simeonov, integral inequality, variant. 
$\alpha \leq n \leq \beta\}$. We use the usual conventions that the empty sums and products are taken to be 0 and 1 respectively. We shall also assume that all the integrals, sums and products involved throughout the discussion exist in the respective domains of their definitions.

Our main result is established in the following theorem.

Theorem 1. Let $U(t), f(t), a(t), b(t)$ be continuous functions on $I=[\alpha, \beta]$, let $a(t)$ and $b(t)$ be nonnegative in $I$, and suppose

$$
U(t) \leq f(t)+\int_{\alpha}^{t} a(s) U(s) d s+\int_{\alpha}^{\beta} b(s) U(s) d s
$$

for $t \in I$. If

$$
p=\int_{\alpha}^{\beta} b(s) \exp \left(\int_{\alpha}^{s} a(\sigma) d \sigma\right) d s<1
$$

then

$$
U(t) \leq f(t)+\frac{1}{1-p} \int_{\alpha}^{\beta} b(s)(f(s)+K(s)) d s \exp \left(\int_{\alpha}^{t} a(\sigma) d \sigma\right)+K(t)
$$

for $t \in I$, where

$$
K(t)=\int_{\alpha}^{t} a(x) f(x) \exp \left(\int_{x}^{t} a(\sigma) d \sigma\right) d x
$$

for $t \in I$.

Proof. Define a function $Z(t)$ by

$$
Z(t)=\int_{\alpha}^{t} a(s) U(s) d s+\int_{\alpha}^{\beta} b(s) U(s) d s .
$$

Then

$$
\begin{gathered}
Z(\alpha)=\int_{\alpha}^{\beta} b(s) U(s) d s \\
U(t) \leq f(t)+Z(t)
\end{gathered}
$$

and

$$
Z^{\prime}(t)=a(t) U(t) \leq a(t) f(t)+a(t) \cdot Z(t) .
$$

Multiplying (8) by the integrating factor $\exp \left(-\int_{\alpha}^{t} a(\sigma) d \sigma\right)$ we have

$$
\frac{d}{d t}\left[Z(t) \exp \left(-\int_{\alpha}^{t} a(\sigma) d \sigma\right)\right] \leq a(t) f(t) \exp \left(-\int_{\alpha}^{t} a(\sigma) d \sigma\right)
$$

By setting $t=x$ in (9) and integrating it with respect to $x$ from $\alpha$ to $t$ we get

$$
Z(t) \exp \left(-\int_{\alpha}^{t} a(\sigma) d \sigma\right)-Z(\alpha) \leq \int_{\alpha}^{t} a(x) f(x) \exp \left(-\int_{\alpha}^{x} a(\sigma) d \sigma\right) d x
$$


which implies

$$
Z(t) \leq Z(\alpha) \exp \left(\int_{\alpha}^{t} a(\sigma) d \sigma\right)+\int_{\alpha}^{t} a(x) f(x) \exp \left(\int_{x}^{t} a(\sigma) d \sigma\right) d x
$$

From (4) and (11) we have

$$
Z(t) \leq Z(\alpha) \exp \left(\int_{\alpha}^{t} a(\sigma) d \sigma\right)+K(t)
$$

Using (12) in (7) we get

$$
U(t) \leq f(t)+Z(\alpha) \exp \left(\int_{\alpha}^{t} a(\sigma) d \sigma\right)+K(t)
$$

Using (13) on the right side of (6) we observe that

$$
\begin{aligned}
Z(\alpha) & =\int_{\alpha}^{\beta} b(s) U(s) d s \\
& \leq \int_{\alpha}^{\beta} b(s)(f(s)+K(s)) d s+Z(\alpha) \int_{\alpha}^{\beta} b(s) \exp \left(\int_{\alpha}^{s} a(\sigma) d \sigma\right) d s
\end{aligned}
$$

From (14) and (2) it follows that

$$
Z(\alpha) \leq \frac{1}{1-p} \int_{\alpha}^{\beta} b(s)(f(s)+K(s)) d s
$$

Using (15) in (13) we get the required inequality in (3).

The discrete analogue of Theorem 1 is given in the following theorem.

Theorem 2. Let $U(n), f(n), a(n), b(n)$ be real-valued function defined on $N_{\alpha, \beta}$, let $a(n)$ and $b(n)$ be nonnegative in $N_{\alpha, \beta}$, and suppose that

$$
U(n) \leq f(n)+\sum_{\sigma=\alpha}^{n-1} a(\sigma) U(\sigma)+\sum_{\sigma=\alpha}^{\beta} b(\sigma) U(\sigma)
$$

for $n \in N_{\alpha, \beta}$. If

$$
r=\sum_{\sigma=\alpha}^{\beta} b(\sigma) \prod_{s=\alpha}^{\sigma-1}(1+a(s))<1
$$

then

$$
U(n) \leq f(n)+\frac{\prod_{s=\alpha}^{n-1}(1+a(s))}{1-r} \sum_{\sigma=\alpha}^{\beta} b(\sigma)(f(\sigma)+K(\sigma))+K(n)
$$


for $n \in N_{\alpha, \beta}$, where

$$
K(n)=\sum_{\sigma=\alpha}^{n-1} a(\sigma) f(\sigma) \prod_{s=\sigma+1}^{n-1}(1+a(s))
$$

Proof. Define a function $Z(n), \alpha \leq n \leq \beta$, by

$$
Z(n)=\sum_{\sigma=\alpha}^{n-1} a(\sigma) U(\sigma)+\sum_{\sigma=\alpha}^{\beta} b(\sigma) U(\sigma) .
$$

Then $Z(\alpha)=\sum_{\sigma=\alpha}^{\beta} b(\sigma) U(\sigma), U(n) \leq f(n)+Z(n)$ and $Z(n+1)-Z(n)=a(n) U(n) \leq$ $a(n) f(n)+a(n) Z(n)$, i.e.

$$
Z(n+1)-(1+a(n)) Z(n) \leq a(n) f(n) .
$$

Multiplying both sides of (20) by $\prod_{s=\alpha}^{n}(1+a(s))^{-1}$, we obtain

$$
Z(n+1) \prod_{s=\alpha}^{n}(1+a(s))^{-1}-Z(n) \prod_{s=\alpha}^{n-1}(1+a(s))^{-1} \leq a(n) f(n) \prod_{s=\alpha}^{n}(1+a(s))^{-1} .
$$

By setting $n=\sigma$ in (21) and taking the sum over $\sigma$ from $\alpha$ to $n-1$ we get

$$
Z(n) \prod_{s=\alpha}^{n-1}(1+a(s))^{-1}-Z(\alpha) \leq \sum_{\sigma=\alpha}^{n-1} a(\sigma) f(\sigma) \prod_{s=\alpha}^{\sigma}(1+a(s))^{-1}
$$

From (22) we have

$$
Z(n) \leq Z(\alpha) \prod_{s=\alpha}^{n-1}(1+a(s))+\sum_{\sigma=\alpha}^{n-1} a(\sigma) f(\sigma) \prod_{s=\sigma+1}^{n-1}(1+a(s))
$$

From (19) and (23) we have

$$
Z(n) \leq Z(\alpha) \prod_{s=\alpha}^{n-1}(1+a(s))+K(n)
$$

for $n \in N_{\alpha, \beta}$.

From (24) and using $U(n) \leq f(n)+Z(n)$ we have

$$
U(n) \leq f(n)+Z(\alpha) \prod_{s=\alpha}^{n-1}(1+a(s))+K(n) .
$$


Using $(25)$ and $Z(\alpha)=\sum_{\sigma=\alpha}^{\beta} b(\sigma) U(\sigma)$ we have

$$
\begin{aligned}
Z(\alpha) & =\sum_{\sigma=\alpha}^{\beta} b(\sigma) U(\sigma) \\
& \leq \sum_{\sigma=\alpha}^{\beta} b(\sigma)(f(\sigma)+K(\sigma))+\sum_{\sigma=\alpha}^{\beta} b(\sigma) Z(\alpha) \prod_{s=\alpha}^{\sigma-1}(1+a(s))
\end{aligned}
$$

From (26) and (17) we have

$$
Z(\alpha) \leq \frac{1}{1-r} \sum_{\sigma=\alpha}^{\beta} b(\sigma)(f(\sigma)+K(\sigma)) .
$$

Using (27) in (25) we get (18) and the proof is complete.

\section{References}

[1] D. Bainov and P. Simenov, Integral Inequalities and Applications, Kluwer Academic Publishers, Dordrecht, 1992.

[2] B. G. Pachpatte, Inequalities for Differential and Integral Equatons, Academic Press, New York, 1998.

[3] B. G. Pachpatte, Inequalities for Finite Difference Equations, Mrcel Dekker Inc., New York, 2001.

Center for General Education, Northern Taiwan Institute of Science and Technology, Peito, Taipei, Taiwan 11202.

Department of Mathematics, Tamkang University, Tamsui, Taiwan 25137. 